\title{
BMJ Open The accuracy of diagnostic Imaging techniques in patients with a suspected Fracture-related Infection (IFI) trial: study protocol for a prospective multicenter cohort study.
}

\author{
GAM Govaert, ${ }^{1}$ MGG Hobbelink, ${ }^{2}$ IHF Reininga, ${ }^{3}$ P Bosch, ${ }^{3}$ TC Kwee, ${ }^{4}$ \\ PA de Jong, ${ }^{2}$ PC Jutte, ${ }^{5}$ HC Vogely, ${ }^{6}$ RAJO Dierckx, ${ }^{7}$ LPH Leenen, ${ }^{1}$ \\ AWJM Glaudemans, ${ }^{7}$ FFA IJpma ${ }^{3}$
}

To cite: Govaert GAM, Hobbelink MGG, Reininga IHF, et al. The accuracy of diagnostic Imaging techniques in patients with a suspected Fracture-related Infection (IFI) trial: study protocol for a prospective multicenter cohort study.. BMJ Open 2019;9:e027772. doi:10.1136/ bmjopen-2018-027772

- Prepublication history and additional material for this paper are available online. To view these files, please visit the journal online (http://dx.doi org/10.1136/bmjopen-2018027772).

Received 08 November 2018 Revised 19 July 2019 Accepted 31 July 2019
Check for updates

(C) Author(s) (or their employer(s)) 2019. Re-use permitted under CC BY-NC. No commercial re-use. See rights and permissions. Published by BMJ.

For numbered affiliations see end of article.

Correspondence to Dr GAM Govaert; g.a.m.govaert@umcutrecht.nl

\section{ABSTRACT}

Introduction The optimal diagnostic imaging strategy for fracture-related infection (FRI) remains to be established. In this prospective study, the three commonly used advanced imaging techniques for diagnosing FRI will be compared. Primary endpoints are (1) determining the overall diagnostic performances of white blood cell (WBC) scintigraphy, fluorodeoxyglucose positron emission tomography (FDG-PET) and magnetic resonance imaging (MRI) in patients with suspected FRI and (2) establishing the most accurate imaging strategy for diagnosing FRI. Methods and analysis This study is a non-randomised, partially blinded, prospective cohort study involving two level 1 trauma centres in The Netherlands. All adult patients who require advanced medical imaging for suspected FRI are eligible for inclusion. Patients will undergo all three investigational imaging procedures (WBC scintigraphy, FDG-PET and MRI) within a time frame of 14 days after inclusion. The reference standard will be the result of at least five intraoperative sampled microbiology cultures, or, in case of no surgery, the clinical presence or absence of infection at 1 year follow-up. Initially, the results of all three imaging modalities will be available to the treating team as per local protocol. At a later time point, all scans will be centrally reassessed by nuclear medicine physicians and radiologists who are blinded for the identity of the patients and their clinical outcome. The discriminative ability of the imaging modalities will be quantified by several measures of diagnostic accuracy. Ethics and dissemination Approval of the study by the Institutional Review Board has been obtained prior to the start of this study. The results of this trial will be disseminated by publication of peer-reviewed manuscripts presentation in abstract form at scientific meetings and data sharing with other investigators through academically established means.

Trial registration number The IFI trial is registered in the Netherlands Trial Register (NTR7490).

\section{INTRODUCTION}

Fracture-related infection (FRI) ${ }^{1}$ is one of the most severe and challenging complications in
Strengths and limitations of this study

- This study is the first to prospectively examine the accuracy of the advanced medical imaging techniques such as white blood cell scintigraphy, fluorodeoxyglucose positron emission tomography and MRI for diagnosing fracture-related infection (FRI).

- The cohort design allows for performing all three investigational imaging modalities in each included patient which will result in a large comparable dataset.

- The multicenter nature of the study will increase external validity of the findings.

- The clinical assessment of the presence of FRI during follow-up could be prone to inaccuracies due to the subjective nature of clinical judgement.

trauma surgery. As with most medical conditions, the key for a successful treatment of FRI is a prompt and accurate diagnosis. Particularly a low-grade FRI, with an often closed wound and little or no physical inflammatory signs, is not easy to recognise.

On average, the incidence of FRI is $1 \%-5 \%^{23}$ with outliers up to $45 \%$ in case of very severe tissue damage and/or contaminated wounds. ${ }^{45}$ The exact number of all fractures that need surgical stabilisation is unknown but it is reported that roughly one-third of all patients with a fracture need hospitalisation and that $80 \%$ of these admitted patients need surgical fracture care. ${ }^{67}$ The incidence of fractures in men is $11.67 / 1000$ / year and in women $10.65 / 1000 /$ year. $^{8}$ This implies that in The Netherlands (the Dutch population in 2018 was roughly 17.2 million) approximately 3000 patients are diagnosed with FRI every year. The number of suspected FRIs is undoubtedly much higher and many 
of these patients require some form of additional diagnostic imaging to confirm or rule out this diagnosis.

FRIs may seem similar to other orthopaedic infections (such as prosthetic joint infections, infected diabetic feet and spondylodiscitis) but there are distinct discrepancies. One of them is that the treatment goal is different: stable fixation and ultimately consolidation of the fracture with the option of implant removal and not, for example, an absolute need for retaining the arthroplasty. Similar differences apply for diagnostic imaging. Regenerating bone and soft tissue healing after surgery and trauma may influence imaging quality and mimic infection. Commonly requested imaging modalities in orthopaedic trauma surgery are conventional X-ray and plain computed tomography (CT). These techniques are particularly good in establishing the degree of fracture healing and the position and integrity of the implants but their accuracy for diagnosing FRI is low. More advanced imaging techniques, such as magnetic resonance imaging (MRI), fluorodeoxyglucose positron emission tomography (FDG-PET) or white blood cell (WBC) scintigraphy are being used to actually diagnose FRI. In the last decade, the use of hybrid camera systems (single photon emission computed tomography (SPECT)-CT, PET-CT or PET-MRI) led to increased precision of the localisation of the infection and, as a consequence, to higher diagnostic accuracy rates. ${ }^{9-11}$

At this moment, no prospective sufficiently powered study has been published on the diagnostic accuracy of medical imaging modalities for FRI. ${ }^{9} 10$ The European Association of Nuclear Medicine (EANM), endorsed by the European Bone and Joint Infection Society (EBJIS), developed guidelines in which the WBC scintigraphy with SPECT/CT is regarded to be the most accurate advanced imaging technique for peripheral bone infection in patients with recent fractures or hardware in situ. ${ }^{12}$ However, this recommendation is based on scarce evidence in the literature and diagnostic studies specifically focussing on FRI are limited and report conflicting outcomes. ${ }^{1013}$ As a result, there are no evidence-based diagnostic guidelines or protocols and a variety of diagnostic strategies depending on local availability and local preference of imaging techniques are being used. ${ }^{14} 15$ These random strategies can lead to unnecessary imaging requests and therefore unnecessary delay of treatment and excessive medical costs.

To resolve this omission, we designed The Accuracy of Diagnostic Imaging Techniques in Patients with a Suspected Fracture-related Infection (IFI) Trial.

\section{Primary endpoints}

1. Determining the overall diagnostic performances of WBC scintigraphy + SPECT/CT, FDG-PET/CT and MRI in patients with suspected FRIs.

\section{Secondary endpoints}

1. Determining whether the accuracy of the different imaging modalities is influenced by patient-related
Table 1 Inclusion and exclusion criteria

\begin{tabular}{|c|c|}
\hline Inclusion criteria & Exclusion criteria \\
\hline Age $\geq 18$ years & Age $<18$ years \\
\hline \multirow{4}{*}{$\begin{array}{l}\text { Suspected FRI according } \\
\text { to the suggestive criteria } \\
\text { of the FRI Consensus } \\
\text { Definition }{ }^{1} \text { (box } 1 \text { ) } \\
\text { requiring advanced } \\
\text { medical imaging }\end{array}$} & $\begin{array}{l}\text { Inability to comply with } \\
\text { study protocol (eg, due to } \\
\text { claustrophobia) }\end{array}$ \\
\hline & $\begin{array}{l}\text { Inability to comply with follow- } \\
\text { up (eg, due to language barrier } \\
\text { or expected loss to follow-up } \\
\leq 1 \text { year) }\end{array}$ \\
\hline & $\begin{array}{l}\text { Known allergies for intravenous } \\
\text { contrast or any of the used } \\
\text { nuclear tracers }\end{array}$ \\
\hline & $\begin{array}{l}\text { No need for advanced imaging } \\
\text { techniques, for example, in case } \\
\text { of early (within } 30 \text { days) surgical } \\
\text { site infection } \\
\text { Pregnant or lactating woman } \\
\text { Uncontrolled diabetes mellitus } \\
\text { type } 1 \text { or } 2\end{array}$ \\
\hline
\end{tabular}

FRI, fracture-related infection.

factors such as in situ implants (plates, screws and intramedullary nails), recent surgery, open wounds, concomitant antibiotic treatment or comorbidities.

2. Determining which imaging modality provides the most valuable information to the surgeon for planning revision surgery, such as the exact location of the infection, the extent of the infection or the presence of sequestra, cloacae, sinus tracts, intracortical or soft tissue abscesses.

3. To assess the quality of life and physical performance of patients with suspected FRIs by using validated patient-reported outcome measures.

4. To design an evidence-based, feasible and cost-effective diagnostic pathway for patients with suspected FRIs.

\section{METHODS AND ANALYSIS}

\section{Study design}

This study is a non-randomised, partially blinded, prospective cohort study involving two level 1 trauma centres in The Netherlands: The University Medical Centre Groningen (UMCG) and the University Medical Centre Utrecht (UMCU). The UMCG is the leading and coordinating centre of this study.

\section{Patient population}

The inclusion and exclusion criteria are presented in table 1. Patients will be recruited at the trauma surgical or orthopaedic outpatient departments, clinical wards and/ or the emergency department of the participating hospitals. All consecutive adult patients in the participating centres, who require advanced medical imaging for 
Box 1 Suggestive and confirmatory fracture-related infection Consensus Definition criteria. ${ }^{1}$

a. Confirmatory criteria

- Presence of a fistula, sinus or wound breakdown (with communication to the bone or implant).

- Phenotypically indistinguishable pathogens identified by culture from at least two separate deep tissue/implant (including sonication-fluid) specimens taken during an operative intervention.

- Presence of microorganisms in deep tissue taken during an operative intervention, as confirmed by histopathological examination using specific staining techniques for bacteria or fungi.

b. Suggestive criteria

- Clinical signs, any one of:

- Pain (without weight bearing, increasing over time, new onset).

- Local redness.

- Local swelling.

- Increased local temperature.

- New onset of joint effusion.

- Fever (single oral temperature measurement of $\geq 38.3^{\circ} \mathrm{C}\left(101^{\circ} \mathrm{F}\right)$ ).

- Radiological signs, any one of:

- Bone lysis (at the fracture site, around the implant).

- Implant loosening.

- Sequestration (occurring over time).

- Failure of progression of bone healing (ie, non-union).

- Presence of periosteal bone formation (eg, at localisations other than the fracture site or in case of a consolidated fracture).

- Elevated serum inflammatory markers in case of a secondary rise (after an initial decrease) or a consistent elevation over a period in time, and after exclusion of other infectious foci or inflammatory processes:

- Erythrocyte sedimentation rate.

- White blood cell count.

- C reactive protein.

- Persistent, increasing or new-onset wound drainage, beyond the first few days postoperatively, without solid alternative explanation.

- A pathogenic organism identified by culture from a single deep tissue/implant (including sonication fluid) specimen taken during an operative intervention.

suspected FRI are eligible for inclusion. Suspected FRI is defined following the clinical suggestive criteria according to the FRI Consensus Definition. ${ }^{1}$ These criteria are based on medical history and clinical examination. Patients with clear signs of acute postoperative surgical site infections within 30 days after their surgery ${ }^{16}$ will be excluded as they usually do not require advanced diagnostic imaging. The suggestive and confirmative FRI Consensus Definition criteria are provided in box 1 .

\section{Interventions}

After enrolment and informed consent, patients are scheduled for undergoing all three investigational imaging procedures (WBC scintigraphy + SPECT/CT, FDG-PET/CT and MRI) within a time frame of 14 days after inclusion (figure 1).

\section{Patient clinical management and follow-up}

Next to the imaging performed, patients will be treated according to the local standard of care. This includes

\section{Suspected Fracture Related Infection}

Inclusion \& signed informed consent

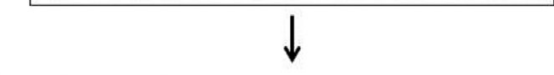

All patients undergo the following medical imaging within 14 days after inclusion

- MRI

- WBC scintigraphy

- FDG-PET
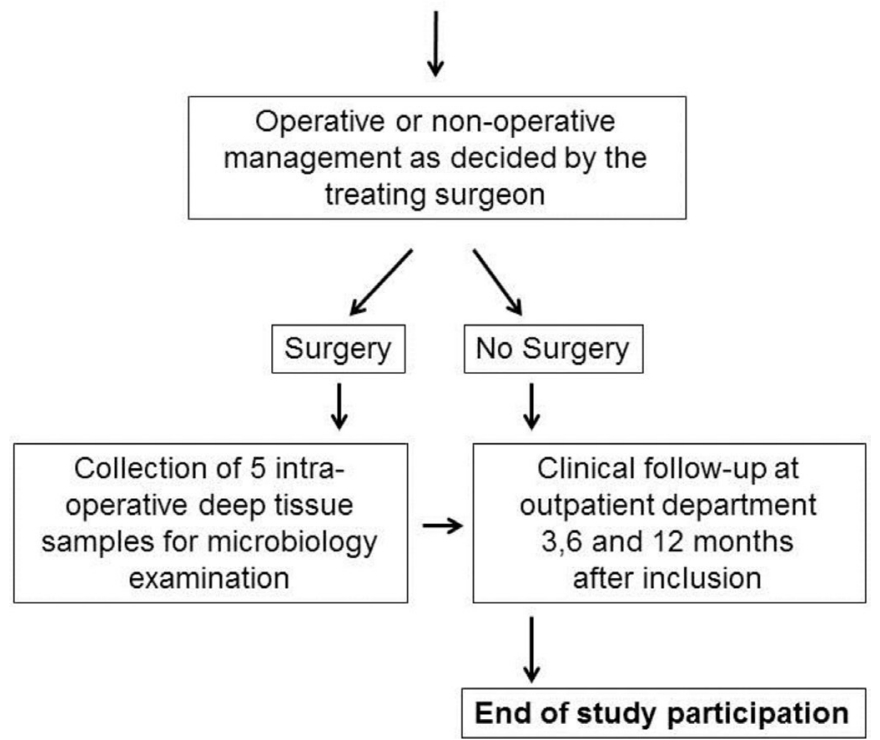

Figure 1 Flowchart of IFI trial. FDG-PET, fluorodeoxyglucose positron emission tomography; FRI, fracture-related infection; MRI, magnetic resonance imaging; IFI, imaging fracture-related infection; WBC, white blood cell.

any additional diagnostic tests, operative treatment, postoperative regimen and postoperative administration of any medication including antibiotics, which will be completely left to the judgement of the treating medical team. The same applies to the decision whether or not to operate based on clinical assessment and imaging. The treating surgeon will be aware of the results of all regular and research imaging procedures performed in this study prior to the decision making moment whether to operate or not.

Included patients who are operated on the clinical suspicion of an FRI will all have adequate tissue sampling for medical microbiology (MMB) examination. Details on the sampling and culturing techniques are discussed in the Reference standard section. The presence or absence of FRI for this group will be judged based on the outcome of the MMB results. To ensure that infection-positive, culture-negative patients are included in the correct final diagnostic group, all included patients will be subjected to the same follow-up regime.

All patients will stay in follow-up for a minimum of 1 year and their clinical status will be assessed by an orthopaedic or trauma surgeon at 3,6 and 12 months (assessments regarding diagnostic criteria for FRI at follow-up are 


\begin{tabular}{|c|c|c|c|c|c|c|c|c|}
\hline & STUDY PERIOD & & & & & & & \\
\hline & Enrolment & & & $\begin{array}{l}\text { Close- } \\
\text { out }\end{array}$ & & & & \\
\hline TIMEPOINT & $-t_{0}$ & $-t_{1}$ & $t_{0}$ & $t_{0}$ & $t_{1}$ & $t_{2}$ & $t_{3}$ & $t_{4}$ \\
\hline Time interval & 0 & $<14$ days & if operation & $\begin{array}{c}\text { if no } \\
\text { operation }\end{array}$ & 3months & $\begin{array}{c}6 \\
\text { months }\end{array}$ & months & 1 year \\
\hline \multicolumn{9}{|l|}{ ENROLMENT: } \\
\hline \multirow{2}{*}{$\begin{array}{l}\text { Eligibility screen } \\
\text { Informed consent }\end{array}$} & $x$ & & & & & & & \\
\hline & $x$ & & & & & & & \\
\hline \multicolumn{9}{|l|}{ INTERVENTIONS: } \\
\hline MRI & & $\mathrm{x}$ & & & & & & \\
\hline FDG-PET/CT & & $x$ & & & & & & \\
\hline $\begin{array}{l}\text { WBC scintigraphy } \\
+S P \text { SCCTCCT }\end{array}$ & & $x$ & & & & & & \\
\hline \multicolumn{9}{|l|}{ ASSESSMENTS: } \\
\hline $\begin{array}{c}\text { Clininal } \\
\text { examintion }\end{array}$ & $x$ & & & & $x$ & $x$ & $x$ & $x$ \\
\hline $\begin{array}{l}\text { Labarotory test } \\
\text { (CRP, LC and ESR) }\end{array}$ & $\mathrm{x}$ & & & & $\mathrm{x}$ & $x$ & $x$ & $x$ \\
\hline $\begin{array}{l}\text { It Itratapopratite } \\
\text { tissue sampling for }\end{array}$ & & & $x$ & & & & & \\
\hline $\begin{array}{r}\text { Serum } \\
\text { inflammation } \\
\text { markers (CRP, LC, }\end{array}$ & $x$ & & & & $x$ & $x$ & $x$ & $x$ \\
\hline 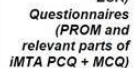 & $x$ & & & & $x$ & $x$ & $\mathrm{x}$ & $x$ \\
\hline
\end{tabular}

Figure 2 Study assessments IFI trial by time point. CRP, C reactive protein; CT, computed tomography; ESR, erythrocyte sedimentation rate; FDG-PET, fluorodeoxyglucose positron emission tomography; IFI, imaging fracture-related infection; iMTA, Institute of Medical Technology assessment; LC, leucocyte count; $M C Q$, medical consumption questionnaire; $\mathrm{PCQ}$, productivity cost questionnaire; PROM, patientreported outcome measure; SPECT, single photon emission computed tomography; WBC, white blood cell.

discussed in the Reference standard section). Standard serum inflammation markers $(\mathrm{C}$ reactive protein (CRP), leucocyte count (LC) and erythrocyte sedimentation rate (ESR)) will be obtained at the time of recruitment and at follow-up (3,6 and 12 months). When the patient visits the outpatient clinic, he/she will be asked to fill out validated patient-reported outcome questionnaires regarding quality of life and physical performance (EuroQol (EQ)5D, Short Musculoskeletal Function Assesment (SMFA)). Health-related productivity losses of paid and unpaid work will be quantified by purchasing relevant parts of the institute of Medical Technology Assessment Productivity Cost Questionnaire (iMTA PCQ). ${ }^{17} 18$ The iMTA Medical Consumption Questionnaire (MCQ) questionnaire and the the Dutch Manual for Costing studies in healthcare will be used to calculate the costs of medical consumption during the research period. ${ }^{19-22}$ The different time points are summarised in figure 2.

\section{Assessment of interventional imaging}

Initially, all imaging is reported on as usual in the participating centres. The written reports will be made available through normal routine procedures and available for the treating physician in the electronic medical patient systems. At a later time point, two board-certified nuclear medicine physicians (for WBC scintigraphy and FDG-PET/CT) and two board-certified radiologists (for MRI), all participating in this trial, will centrally reassess all scans. This is done to obtain uniformity when interpreting the results. Imaging interpretation criteria are provided in the online supplemental material.

All scans will be encoded so that the central reviewers will be blinded for the identity of the patient and their clinical outcome. Case report forms (CRF) are developed to score each scan technique. If the two observers disagree, a third reader will review the images and the final classification will be made in consensus. The scans will finally be classified as 'negative for FRI' or 'positive for FRI'. The reports will also focus on specific anatomic features such as the presence of fistulas, sequesters and soft tissue involvement/abscesses. In case of surgery, surgeons are requested to fill out a CRF on the operative findings to match these with the anatomic features of the preoperative scans.

\section{Imaging protocols}

Preparation, administration, acquisition and interpretation of all imaging techniques will be performed according to the existing guidelines of the $\mathrm{EANM}^{2324}$ and the American College of Radiology ${ }^{25}$; details are provided in the online supplementary material.

\section{Reference standard}

The reference standard for determining the diagnostic accuracy of the WBC scintigraphy, the FDG-PET/CT and MRI will be the presence of FRI according to the FRI Consensus Definition ${ }^{1}$. For the patients who are not operated, the reference standard will be the presence of FRI during follow-up. For this purpose, a board-certified trauma or orthopaedic surgeon will record all clinical findings (based on the consensus definition criteria, box $1(\mathrm{a}$ and $\mathrm{b})$ ) at 3,6 and 12 months follow-up on a predesigned Case Report Form (CRF). The clinical presence or absence of infection will subsequently be judged according to the information provided on the CRF and based on the FRI Consensus Definition by two blinded reviewers who are not involved in the actual care of the patient. In all patients who are operated on at least five surgical bone, implant and/or tissue samples will be obtained for microbiology culturing. These cultures will be sampled in a protocolled manner with clean surgical instruments for each sample to avoid cross contamination. ${ }^{26}$ Preferably all antibiotics are discontinued for at least 2 weeks and antibiotic prophylaxis is withheld until the last sample is obtained. ${ }^{27} 28$ The criteria for a positive result based on operatively obtained cultures are listed in box 1 (a). To ensure that infection-positive, culture-negative patients are included in the correct final diagnostic group, all patients will be subjected to the same follow-up regime. In case of (revision) surgery during follow-up the same sampling protocol will be followed. Intermittent suggestive symptoms such as local pain or redness (with or without 'treatment' with antibiotics) alone are not regarded confirmative for FRI. They can however prompt the surgeon to order diagnostic tests followed by an operation and tissue sampling, which can subsequently lead to positive microbiology results. 


\section{Collection of additional medical data}

For all patients, information concerning patient characteristics (age, gender, body mass index), social and working context ( $\mathrm{T}=0$; validated quality of life, functional outcome and healthcare cost questionnaires), medication (non steroid anti-inflammatory drugs (NSAID's), immunosuppressive drugs, antibiotics, painkillers), nicotine/ substance abuse, comorbidities (eg, diabetes), history of trauma/fracture, type of implants being used (plates, screws and intramedullary nails) in previous operations, clinical presentation of FRI according to the criteria of the consensus definition, ${ }^{1}$ laboratory results (ESR, LC, CRP), X-ray findings (fracture union, implant failure), annotation of the indication for which the physician prompted additional imaging, patient's opinion about the preference and tolerance regarding each imaging modality, operation records (type, osteosynthesis insitu, macroscopic signs of infection according to the Cierny-Mader and B.A.C.H. classification, ${ }^{29}{ }^{30}$ abscess, sequestrum, involucrum, cloacae, sinus tract, fistula or septic artritis according to a standardised CRF), microbiology results (number of deep tissue cultures, pathogens), antibiotic treatment and follow-up data at 3, 6 and 12 months (concerning clinical findings based on the consensus definition criteria together with validated quality of life, functional outcome and healthcare cost questionnaires) will be recorded.

\section{Establishing a diagnostic pathway for patients with suspected FRI}

Based on the results of this trial, a diagnostic pathway for patients with suspected FRI will be established. In this pathway, the use of the imaging modality or a combination of different imaging modalities with the highest accuracy and feasibility will be described. The costs of the different imaging modalities will be taken into account. For these costs, standard unit prices and rates that are included in the Manual for Cost Analyses, Methods and Standard Prices for Economic Evaluation in Health Care will be used. ${ }^{20}$

\section{Sample size and power}

Recently, we performed a systematic review about the accuracy of diagnostic imaging modalities for peripheral post-traumatic osteomyelitis (the old terminology for FRI).$^{10}$ Based on the best available evidence over the last 16 years, the sensitivity for WBC scintigraphy ranged from $50 \%$ to $100 \%$, and specificity ranged from $40 \%$ to $97 \%$. For FDG-PET, this was $83 \%-100 \%$ and $51 \%-100 \%$, and for MRI $82 \%-100 \%$ and $43 \%-60 \%$, respectively. Moreover, studies which combined the WBC scintigraphy with SPECT/CT or the FDG-PET with a CT scan showed some increase in diagnostic accuracy measures. Overall, our review demonstrated that sensitivity and specificity vary widely between studies, because the literature about FRIs is limited and hampered by small case series, heterogeneous patient populations, different imaging acquisition and interpretations protocols and quickly evolving (combined) imaging techniques. Another recent publication from our group has shown that the sensitivity and specificity of WBC scintigraphy to diagnose FRI in a large retrospectively analysed patient cohort is 0.86 and 0.98 , respectively. ${ }^{31}$ In two other recent retrospective studies on the accuracy of FDG-PET/CT sensitivities and specificities between 0.65 and 0.89 and 0.77 and 0.80 are reported, respectively. ${ }^{32} 33$ Based on these results we expect a difference of at least $15 \%$ in sensitivity and specificity between the WBC scintigraphy and the FDG-PET/CT. The accuracy of MRI for FRI is reported to be even lower than that of the FDG-PET $/ \mathrm{CT}^{10}$ so this expected difference will also apply for this modality.

Sensitivity refers to the percentage of patients who are correctly identified as having the disease, in this case an FRI. With a sample size of $n=50$ (patients who actually have an FRI) it is possible to detect a statistically significant difference between the sensitivity of WBC scintigraphy (sensitivity of 0.86 ) and the other diagnostic modalities (maximal expected sensitivity of 0.71 ): $95 \%$ CI around the sensitivity of 0.71 will be 0.58 to 0.84 . Since the sensitivity of WBC scintigraphy of 0.86 is outside this $95 \% \mathrm{CI}$, it should be considered statistically significant relative to the sensitivity of the other diagnostic modalities.

Specificity refers to the percentage of patients who are correctly identified as not having the disease, in this case an FRI. With a sample size of $n=120$ (patients who do not have an FRI) it is possible to detect a statistically significant difference between the specificity of WBC scintigraphy (specificity of 0.98 ) and the other diagnostic modalities (maximal expected specificity of 0.83 ); $95 \%$ CI around the specificity of 0.83 will be 0.75 to 0.91 . Since the specificity of WBC scintigraphy of 0.98 is outside this $95 \% \mathrm{CI}$, it is statistically significant different relative to the specificity of the other diagnostic modalities.

In the aforementioned study on the accuracy of WBC scintigraphy for diagnosing FRI, ${ }^{31}$ it was established that approximately $30 \%$ of the patients who undergo diagnostic imaging for suspected FRI actually have an FRI. The study sample thus needs to contain 170 patients, of whom 50 patients are expected to have an FRI (needed for the sensitivity calculation), and 120 patients are expected to not have an FRI (needed for the specificity calculation). To correct for loss-to-follow-up of approximately $20 \%$, a total sample size of $n=200$ is needed.

\section{Feasibility}

We anticipate that we will be able to recruit a sufficient number of patients during the study period. Both contributing hospitals in this multicentre cohort study are level 1 trauma centres with 50-75 patients suffering from FRI each year. We assume that at least half of the eligible patients are willing to participate in this study. This assumption is based on our experience that most trauma patients are eager to comply with additional diagnostic imaging in case of a suspected FRI. This could be due to the fact that they suffer from a long-standing disease and might feel underdiagnosed. Therefore, we 
anticipate including 50-60 patients a year in this study. This combined with a follow-up period of 1 year will make it feasible to complete this study in 5 years.

\section{Statistical analysis}

Summary statistics and analyses will be provided for all patients who undergo WBC scintigraphy + SPECT/CT, FDG-PET/CT and MRI. The discriminative ability of the imaging modalities will be quantified by several measures of diagnostic accuracy: sensitivity/specificity (including receiver operating characteristics (ROC) curves), positive and negative predictive values, positive and negative likelihood ratios, and the diagnostic odds ratio (OR). To assess whether a variable (such as type of implants, recent surgery, open wounds, NSAID use or concomitant antibiotic treatment) is predictive of a false imaging test result (false-positive or false-negative vs true-positive or true-negative), multivariable logistic regression analysis will be performed.

\section{Patient and public involvement}

No patients were involved in the design of this study. This decision was based on the fact that in a previous patient participating meeting for the Dutch guideline 'diagnosis and treatment of FRI' in which two authors (GG, AWJMG) of the current study were involved, no concerns regarding medical imaging were reported by any of the patients. However, patient-reported outcome questionnaires regarding quality of life and physical performance (EQ-5D, SMFA) will be part of the data collected in this study.

\section{TRIAL STATUS}

The IFI trial has been open for recruitment since July 2019 in the UMCG, the UMCU will follow by the end of this year. To date five patients have been included. Estimated study duration is 60 months with a completion date in July 2024.

The IFI Trial is registered in the Netherlands Trial Register (NTR7490). Registration date: 16-09-2018. Website: www.trialregister.nl

\section{ETHICS AND DISSEMINATION \\ Patient safety}

Use of radiopharmaceuticals means exposure to ionising radiation. Because of the potential hazards of radiation, guidelines for the exposure of healthy volunteers and patients in The Netherlands are specified in Besluit Stralingsbescherming (BS 2000), artikel 60, Staatsblad 2001, 397 according to the guidelines of the International Commission on Radiological Protection. The extra imaging modality (depending on site specific common practice in collaborating centres) will be the MRI and either the WBC scintigraphy or the FDG-PET/CT. Overall, the additional radiation exposure for a patient participating in this trial will be $4.0 \mathrm{mSv}$. This complies with ICRP 62, category IIb, which means it is justified in patients.

\section{Data and safety monitoring board (DSMB) and interim analysis}

All research modalities are well-established, common and safe procedures used in clinical practice for this patient population every day. Therefore, the risk of additional adverse events will be minimal. This means that there will be no need to implement a DSMB or interim analysis.

\section{Dissemination plan}

The results of this trial will be disseminated by publication of peer-reviewed manuscripts, presentation in abstract form at scientific meetings and data sharing with other investigators through academically established means. The outcome of this trial will also be utilised to design an evidence-based, feasible and cost-effective diagnostic pathway for patients with suspected FRI and implement this in national and international guidelines.

\section{Data storage and management}

All data will be entered by site principal investigators (PIs) or research assistants and data accuracy will be verified by the study PI. Data quality control measures will include queries to identify missing data, outliers and discrepancies. Only research assistants and site PIs will have access to protected health information. After enrolment, a unique identifier will be assigned to each study subject. The data from all sites will be uploaded and stored using a web-based data management application. All computers will be password protected and encrypted per university policy. The PI will ensure that the anonymity is maintained. Patients will not be identified by name in any reports on this study. The study PI will have access to the final study dataset.

\section{Author affiliations}

${ }^{1}$ Department of Trauma Surgery, Utrecht University, University Medical Center Utrecht, Utrecht, The Netherlands

${ }^{2}$ Department of Radiology and Nuclear Imaging, Utrecht University, University Medical Center Utrecht, Utrecht, The Netherlands

${ }^{3}$ Department of Trauma Surgery, University of Groningen, University Medical Center Groningen, Groningen, The Netherlands

${ }^{4}$ Department of Radiology, University of Groningen, University Medical Center Groningen, Groningen, The Netherlands

${ }^{5}$ Department of Orthopaedic Surgery, University of Groningen, University Medical Center Groningen, Groningen, The Netherlands

${ }^{6}$ Department of Orthopaedic Surgery, Utrecht University, University Medical Center Utrecht, Utrecht, The Netherlands

${ }^{7}$ Department of Nuclear Medicine and Molecular Imaging, University of Groningen, University Medical Center Groningen, Groningen, The Netherlands

Contributors All authors were actively involved in this study design and read and approved the final manuscript.GG, IR, AG and FIJ initiated and designed this study. $A G, M H, P d J, R D$ and TK were responsible for drafting the imaging protocols and resolving the necessary logistic challenges. IR performed the power analysis and will conduct the statistical analysis. PB, PCJ, HV and LL participated in the critical appraisal of the study design and this manuscript.

Funding This trial is financially supported by grant funding from DePuySynthes, subject to and conditional upon execution of a research funding agreement (DPS-TCMF-2019-014). 
Competing interests None declared.

Patient consent for publication Not required.

Ethics approval The Institutional Review Board of the leading and coordinating centre (the UMCG) approved this study (number METc 2018/141). This study will be conducted according to the principles of the Declaration of Helsinki and in accordance with the Medical Research involving Human Subjects Act.

Provenance and peer review Not commissioned; externally peer reviewed.

Open access This is an open access article distributed in accordance with the Creative Commons Attribution Non Commercial (CC BY-NC 4.0) license, which permits others to distribute, remix, adapt, build upon this work non-commercially, and license their derivative works on different terms, provided the original work is properly cited, appropriate credit is given, any changes made indicated, and the use is non-commercial. See: http://creativecommons.org/licenses/by-nc/4.0/.

\section{REFERENCES}

1. Metsemakers WJ, Morgenstern M, McNally MA, et al. Fracturerelated infection: a consensus on definition from an international expert group. Injury 2018;49:505-10.

2. Ochsner EBO, Bodler P-M, Broger I. Infections of the musculoskeletal system. In: 1 ed: Swiss orthopaedics in-house publisher, Grandvaux. Switzerland, 2014: 260.

3. Renz N, Feihl S, Dlaska CE, et al. [Osteosynthesis-associated infections : Epidemiology, definition and diagnosis]. Unfallchirurg 2017;120:454-60.

4. Marsh JL, Smith ST, Do TT. External fixation and limited interna fixation for complex fractures of the tibial plateau. J Bone Joint Surg Am 1995;77:661-73.

5. Henkelmann R, Frosch $\mathrm{K}-\mathrm{H}$, Glaab R, et al. Infection following fractures of the proximal tibia - a systematic review of incidence and outcome. BMC Musculoskelet Disord 2017;18:481.

6. Singer BR, McLauchlan GJ, Robinson CM, et al. Epidemiology of fractures in 15,000 adults: the influence of age and gender. $J$ Bone Joint Surg Br 1998;80:243-8.

7. Somersalo A, Paloneva J, Kautiainen $\mathrm{H}$, et al. Incidence of fractures requiring inpatient care. Acta Orthop 2014;85:525-30.

8. Court-Brown CM, Caesar B. Epidemiology of adult fractures: a review. Injury 2006;37:691-7.

9. Govaert GAM, Glaudemans AWJM. Nuclear medicine imaging of posttraumatic osteomyelitis. Eur J Trauma Emerg Surg 2016;42:397-410.

10. Govaert GA, IJpma FF, McNally M, et al. Accuracy of diagnostic imaging modalities for peripheral post-traumatic osteomyelitis - a systematic review of the recent literature. Eur J Nucl Med Mol Imaging 2017;44:1393-407.

11. Glaudemans AW, Prandini N, DI Girolamo M, et al. Hybrid imaging of musculoskeletal infections. Q J Nucl Med Mol Imaging 2018;62:3-13.

12. Glaudemans AWJM, Jutte PC, Cataldo MA, et al. Consensus document for the diagnosis of peripheral bone infection in adults: a joint paper by the EANM, EBJIS, and ESR (with ESCMID endorsement). Eur J Nucl Med Mol Imaging 2019;46:957-70.

13. Termaat MF, Raijmakers PGHM, Scholten HJ, et al. The accuracy of diagnostic imaging for the assessment of chronic osteomyelitis: a systematic review and meta-analysis. J Bone Joint Surg Am 2005;87:2464-71.

14. Govaert GAM, Glaudemans AWJM, Ploegmakers JJW, et al. Diagnostic strategies for posttraumatic osteomyelitis: a survey amongst Dutch medical specialists demonstrates the need for a consensus protocol. Eur J Trauma Emerg Surg 2018;44:417-26.
15. Jutte P, Lazzeri E, Sconfienza LM, et al. Diagnostic flowcharts in osteomyelitis, spondylodiscitis and prosthetic joint infection. Q J Nucl Med Mol Imaging 2014;58:2-19.

16. Procedure-associated Module SSI. Centers for disease control and prevention (CDC), 2017. Available: https://www.cdc.gov/nhsn/pdfs/ pscmanual/9pscssicurrent.pdf [Accessed 15 mar 2015].

17. Bouwmans $\mathrm{C}$, Krol M, Severens $\mathrm{H}$, et al. The iMTA productivity cost questionnaire: a standardized instrument for measuring and Valuing health-related productivity losses. Value Health 2015;18:753-8.

18. Bouwmans C, Hakkaart-van Roijen L, Koopmanschap M, et al. iMTA Productivity Cost Questionnaire [in Dutch], 2013. Available: https://www.solkweb.nl/wp-content/uploads/2017/04/iPCQpdf.pdf [Accessed 18 Jul 2018].

19. Bouwmans C, Hakkaart-van Roijen L, Koopmanschap M, et al. iMTA Medical Consumption Questionnaire [in Dutch], 2013. Available: https://www.solkweb.nl/wp-content/uploads/2017/04/iMCQ.pdf [Accessed 18 Jul 2018].

20. Kanters TA, Bouwmans CAM, van der Linden N, et al. Update of the Dutch manual for costing studies in health care. PLoS One 2017;12:e0187477.

21. Versteegh M, Knies S, Brouwer W. From good to better: new Dutch guidelines for economic evaluations in healthcare. Pharmacoeconomics 2016;34:1071-4.

22. Nederland Z. Guideline for conducting economic evaluations in healthcare [in Dutch: Richtlijn voor het uitvoeren van economische evaluaties in de gezondheidszorg], 2016.

23. Signore $A$, Jamar $F$, Israel $O$, et al. Clinical indications, image acquisition and data interpretation for white blood cells and antigranulocyte monoclonal antibody scintigraphy: an EANM procedural guideline. Eur J Nucl Med Mol Imaging 2018;45:1816-31.

24. Jamar F, Buscombe J, Chiti A, et al. EANM/SNMMI guideline for 18F-FDG use in inflammation and infection. J Nucl Med 2013;54:647-58.

25. Beaman FD, Kransdorf MJ, Kransdorf MJ, et al. ACR Appropriateness Criteria ${ }^{\circledR}$ Suspected Osteomyelitis, Septic Arthritis, or Soft Tissue Infection (Excluding Spine and Diabetic Foot). J Am Coll Radiol 2017;14:S326-S37.

26. Hellebrekers P, Rentenaar RJ, McNally MA, et al. Getting it right first time: the importance of a structured tissue sampling protocol for diagnosing fracture-related infections. Injury 2019. doi:10.1016/j. injury.2019.05.014. [Epub ahead of print: 07 Jun 2019].

27. Atkins BL, Athanasou N, Deeks JJ, et al. Prospective evaluation of criteria for microbiological diagnosis of prosthetic-joint infection at revision arthroplasty. The Osiris collaborative Study Group. J Clin Microbiol 1998;36:2932-9.

28. Dudareva M, Barrett L, Figtree $M$, et al. Sonication versus tissue sampling for diagnosis of prosthetic joint and other orthopedic device-related infections. J Clin Microbiol 2018;56. doi:10.1128/ JCM.00688-18

29. Cierny G, Mader JT, Penninck JJ. The classic: a clinical staging system for adult osteomyelitis. Clin Orthop Relat Res 2003;414:7-24.

30. Hotchen AJ, Sendi P, McNally M. Bach: a new classification system for long-bone osteomyelitis. Available: https:// onlineboneandjointorguk/doi/abs/101302/1358-992X201722055 [Accessed 22 may 2019].

31. Govaert GAM, Bosch P, IJpma FFA, et al. High diagnostic accuracy of white blood cell scintigraphy for fracture related infections: results of a large retrospective single-center study. Injury 2018;49:1085-90.

32. van Vliet KE, de Jong VM, Termaat MF, et al. Fdg-Pet/Ct for differentiating between aseptic and septic delayed union in the lower extremity. Arch Orthop Trauma Surg 2018;138:189-94.

33. Lemans JVC, Hobbelink MGG, IJpma FFA, et al. The diagnostic accuracy of ${ }^{18} \mathrm{~F}-\mathrm{FDG} \mathrm{PET} / \mathrm{CT}$ in diagnosing fracture-related infections. Eur J Nucl Med Mol Imaging 2019;46:999-1008. 